\title{
EVALUACIÓN FORMATIVA Y COMPARTIDA EN LOS PROYECTOS DE TRABAJO TUTORADO, UN EJEMPLO DE BUENA PRÁCTICA
}

Formative and shared assessment in tutored learning projects, an example of good practice

Avaliação formativa e compartilhada em projetos de trabalho tutorados, um exemplo de boas praticas

Apoyo/financiación del trabajo: Este estudio se ha llevado a cabo dentro del proyecto de $\mathrm{I}+\mathrm{D}+\mathrm{i}:$ "La competencias docentes en la formación inicial del profesorado de educación física”. Convocatoria de noviembre de 2013 del Programa Estatal de Investigación, Desarrollo e Innovación Orientada a los Retos de la Sociedad, en el marco del Plan Estatal de Investigación Científica y Técnica y de Innovación 2013-2016. Referencia: EDU 2013-42024-R. Duración: 3 años (2014-2016).

\section{Raúl A. Barba-Martín (1)}

\section{Víctor M. López-Pastor (2)}

(1) Facultad de Educación de Segovia (Universidad de Valladolid), Segovia España. Teléfono: +34 921112321. Correo electrónico: raulalberto.barba@uva.es

(2) Facultad de Educación de Segovia (Universidad de Valladolid), Segovia, España. Teléfono: +34 921112321. Correo electrónico: vlopez@pdg.uva.es

\section{Resumen}

En este trabajo se presenta una experiencia de buena práctica de aprendizaje y de evaluación formativa y compartida, llevada a cabo en Formación Inicial del Profesorado (FIP). Nos centramos en el papel que juega la metodología de "Proyectos de Aprendizaje Tutorado", utilizado en cuarto curso de FIP, en la titulación de maestro de Educación Infantil, durante el primer cuatrimestre del curso 2016-2017, en la asignatura de “Expresión y comunicación corporal”. Los resultados han sido muy positivos, tanto en ventajas encontradas por parte del alumnado con la metodología de aprendizaje y el sistema de evaluación utilizado, como en satisfacción general y en la percepción de competencia adquirida durante la asignatura. Además, se obtienen valores muy altos en 
rendimiento y éxito académico. Se ha medido también la carga de trabajo del alumnado, que está dentro de las horas oficialmente previstas de trabajo no presencial.

Palabras clave: Evaluación Formativa; Evaluación Compartida; Formación Inicial del Profesorado; Educación Infantil; Proyecto de Aprendizaje Tutorado

\begin{abstract}
This paper presents an experience of good learning practice and of formative and shared assessment, carried out in Pre-service Teacher Education (PTE). We focus on the role of the "Tutored Learning Projects" methodology, used in the fourth PTE course, in the qualification of Early Childhood Teacher, during the first semester of the 2016-2017 course, in the subject of "Expression and Body Communication ". The results have been very positive, both in the advantages found by the students with the learning methodology and the assessment system used, as well as in general satisfaction and in the perception of competence acquired during the subject. Very high values are also obtained in performance and academic success. It has also measured the workload of the students, which is within the officially planned hours of non-face-to-face work.
\end{abstract}

Keywords: Formative Assessment; Shared Assessment; Pre-service Teacher Education; Kindergarden; Tutored Learning Projects

\title{
Resumo
}

Este artigo apresenta uma experiência de aprendizagem boa prática e formativa e avaliação compartilhada, realizado em Formação Inicial de Professores (FIP). Nós nos concentramos sobre o papel da metodologia de "Projetos Explicações de Aprendizagem", utilizados no quarto ano do FIP, mestrado em Educação Infantil, durante o primeiro semestre do curso de 2016-2017 no assunto "Expressão e comunicação do corpo. " Os resultados têm sido muito positivos, tanto vantagens encontradas pelos alunos com metodologia de aprendizagem e sistema de avaliação utilizado, e satisfação geral e percepção de competência adquiridos durante o curso. valores muito elevados também são obtidas no desempenho e sucesso acadêmico. Ele também mediu a carga de trabalho dos alunos, o que está dentro das horas oficialmente programadas de trabalho sem contato.

Palavras-chave: Avaliação Formativa; Avaliação Compartilhada; Formação Inicial de Professores; Educação Infantil; Projeto de Aprendizagem Tutorado 


\section{Introducción}

En el centro llevamos muchos años trabajando con los Proyectos de Aprendizaje Tutorado (PAT), porque han demostrado ser una forma muy eficaz de trabajo teóricopráctico y de desarrollo de competencias profesionales. Se trata de una actividad de aprendizaje grupal. Cada grupo tiene que preparar una sesión práctica y un marco teórico a elegir dentro de una lista de temas y propuestas de intervención que se ofrecen a la clase a principio de curso. En el apartado de diseño explicamos las fases a seguir. El proceso de evaluación formativa y compartida durante las tutorías es clave a la hora de lograr mejores aprendizajes y de asegurar una buena calidad de los PAT.

\section{Contextualización}

La experiencia se ha llevado a cabo en la Facultad de Educación de Segovia (Universidad de Valladolid), en cuarto curso de grado de "Maestro de Educación Infantil”, durante el primer cuatrimestre del curso 2016-2017, en la asignatura de “Expresión y comunicación corporal”. Se trata de una asignatura de 6 créditos, en la que cada alumno recibe unas 60 horas lectivas, a las que hay sumar 90 de trabajo no presencial. Se trabaja con dos grupos, el primero de 42 alumnas, que sí que desdobla en las horas prácticas, y un segundo grupo de otra mención, de sólo 10 alumnos y alumnas, que no desdobla.

\section{Diseño y desarrollo}

El proceso de elaboración de cada PAT es el siguiente:

1-Se asigna una fecha de realización para cada grupo.

2-En tutorías se explica a cada grupo los documentos básicos que tienen que leer sobre cada propuesta.

3-El grupo elabora un borrador del marco teórico (4 páginas) y un plan de sesión y acuden a una tutoría grupal, donde el profesor corrige los borradores y les dice las cosas que deben ser mejoradas y corregidas.

4-Este proceso se repite hasta que los dos documentos tienen una calidad aceptable.

5-Se realiza la sesión práctica en la fecha elegida y a continuación se expone el marco teórico en 10 minutos, que ha sido previamente subido a la web, para que todo el alumnado se lo pueda descargar y tenerlo. 
6-Adaptan el PAT para un grupo real de niños de educación infantil, en uno de los colegios de la ciudad, y lo realizan dentro del horario lectivo.

7-Una semana después deben entregar el informe final del PAT en el que reflexionan sobre las dos puestas en práctica de su trabajo. Si está correcto el profesor se lo devuelve para que lo guarden en su carpeta. Si hay aspectos a mejorar deben corregirlo y volverlo a entregar en un plazo máximo de una semana.

\section{Evaluación}

En la tabla 1 presentamos un resumen de las actividades de aprendizaje, con los procesos de evaluación asociada a cada una, así como el peso que tiene en la calificación final.

Tabla 1.

Resumen de actividades de aprendizaje, evaluación y peso en la calificación final.

\begin{tabular}{lllc}
\hline Actividad de aprendizaje & Instrumento evaluación & Evaluación formativa & $\begin{array}{c}\text { Peso en } \\
\text { calificación }\end{array}$ \\
\hline 1-PAT-proyecto de & -Escala descriptiva & En todos los casos cada & 35 \\
aprendizaje tutorado & (rúbrica) & documento entregado se & \\
2-Fichas de sesiones & -Escala descriptiva & revisa por el profesorado, & 20 \\
prácticas & (rúbrica) & aportando feed-back para & 20 \\
3-Examen parcial con co- & -Planilla & su mejora. & \\
evaluación inmediata & & Si hay cosas que tengan & 10 \\
\hline 4-Mapas conceptuales y & -Escala descriptiva & que ser corregidas, se da & \\
dossier asignatura & (rúbrica) & una semana de plazo para & \\
\cline { 4 - 4 } 5-Lecturas y tertulias & -Escala descriptiva & hacerlo & \\
dialógicas & (rúbrica) & & \\
\hline
\end{tabular}

Tanto la "buena práctica” como la asignatura en su conjunto se evalúan a través de de varios instrumentos estandarizados que ha ido generando la Red de Evaluación Formativa, al igual que en todos los informes de “buenas prácticas” recogidos cada curso: (a) cuestionario anónimo de evaluación (para el alumnado), que utiliza una escala tipo likert de 1-5; (b) escala de autopercepción de competencia del alumnado, que utiliza una escala tipo likert 1-4

\section{Conclusiones}

La experiencia recibe una valoración muy positiva por parte del alumnado de la asignatura; las medias de todos los ítems están entre 3.7 y 4.7 (escala 1-5), tanto en los 
ítems sobre la buena práctica como en los de la asignatura en su conjunto. En lo que respecta al cuestionario de autopercepción de competencia, la mayoría de los ítems tienen valoraciones altas en la mayoría de las competencia transversales, así como las específicas de educación física en edades tempranas, entre 3 y 3.50 (escala 1-4).

La tasa de éxito es muy alta (96,1\%), con un buen rendimiento académico en la mayoría del alumnado, efecto lógico de una alta implicación y trabajo durante todo el proceso de aprendizaje. La carga media de trabajo del alumnado es de 85 horas, por lo que se ajusta muy bien a la carga no lectiva prevista (90 horas). Por todo ello la valoración de la experiencia es muy positiva. 